Michael J. Evans, Department of Mathematics, Washington and Lee University, Lexington, VA 24450, email: mjevans@@wlu.edu

Richard J. O'Malley, Department of Mathematical Sciences, University of Wisconsin - Milwaukee, WI 53201, email: omalley@@csd.uwm.edu

\title{
FINE TUNING THE RECOVERABILITY OF BAIRE ONE FUNCTIONS*
}

\begin{abstract}
It is known that a real-valued function on the unit interval belongs to Baire class one if and only if it is first return recoverable. Here it is shown that the trajectory used for recovering the function can be modified in such a way as to reflect local behavior of the function.
\end{abstract}

\section{Introduction}

Several standard subcollections of the class of real-valued Baire one functions defined on $[0,1]$ have been characterized utilizing first return limiting notions. For example, for a function $f:[0,1] \rightarrow \mathbb{R}$ it is known that $f$ is Baire one if and only if $f$ is first return recoverable [3], that $f$ is Baire one and has a dense in itself graph if and only if $f$ is first return approachable [1], and that $f$ is Baire one, Darboux if and only if $f$ is first return continuous [2]. Here we will take a close look at what can be said about the trajectories involved in these characterizations. In particular, we want to observe that a trajectory can be chosen in such a way as to closely mirror the local behavior of the function. For example, if $f$ is a Baire one function, we want to show that the trajectory can be chosen in such a way that if $(x, f(x))$ is isolated from neither the left nor right in the graph of $f$, then $f$ will not only be first return recoverable at

\footnotetext{
Key Words: first return continuous, first return approachable, first return recoverable, Baire class one

Mathematical Reviews subject classification: Primary: 26A03; Secondary: 26A21, $26 \mathrm{~A} 15$

Received by the editors September 14, 1994

* The authors are on the Editorial Board of the Real Analysis Exchange. This paper was managed by another member of the Editorial Board.
} 
$x$ with respect to this trajectory, but actually first return continuous at $x$ with respect to this trajectory. We shall demonstrate similar behavior for situations where $(x, f(x))$ is isolated on one side, obtaining first return approachability at such a point. The next section contains the definitions of the terminology used in these remarks.

\section{First Return Terminology and Notation}

By a trajectory we mean any sequence $\left\{x_{n}\right\}_{n=0}^{\infty}$ of distinct points in $[0,1]$, which is dense in $[0,1]$. One method of specifying a trajectory is to assign an enumeration or ordering to a given countable dense subset $D$ of $[0,1]$. We shall refer to such a set $D$ as a support set.

Let $\left\{x_{n}\right\}$ be a fixed trajectory. For a given interval, or finite union of intervals, $H \subseteq[0,1], r(H)$ will be the first element of the trajectory $\left\{x_{n}\right\}$ in $H$. First, for $x \in[0,1]$ we define what we shall mean by the first return route to $x$ based on the trajectory $\left\{x_{n}\right\}$. If $\rho>0$, we use $B_{\rho}(x)$ to denote $\{y \in[0,1]:|x-y|<\rho\}$. The first return route to $x, \mathcal{R}_{x}=\left\{y_{k}\right\}_{k=1}^{\infty}$, is defined recursively via

$$
\begin{gathered}
y_{1}=x_{0}, \\
y_{k+1}= \begin{cases}r\left(B_{\left|x-y_{k}\right|}(x)\right) & \text { if } x \neq y_{k} \\
y_{k} & \text { if } x=y_{k} .\end{cases}
\end{gathered}
$$

We say that $f:[0,1] \rightarrow \mathbb{R}$ is first return recoverable with respect to $\left\{x_{n}\right\}$ at $x$ provided that

$$
\lim _{k \rightarrow \infty} f\left(y_{k}\right)=f(x)
$$

and if this happens for each $x \in[0,1]$, we say that $f:[0,1] \rightarrow \mathbb{R}$ is first return recoverable with respect to $\left\{x_{n}\right\}$. Further, we say that $f$ is first return recoverable if there exists a trajectory $\left\{x_{n}\right\}$ such that $f$ is first return recoverable with respect to $\left\{x_{n}\right\}$.

For $0<x \leq 1$, the left first return path to $x$ based on $\left\{x_{n}\right\}, \mathcal{P}_{x}^{l}=\left\{t_{k}\right\}$, is defined recursively via

$$
t_{1}=r(0, x) \text {, and } t_{k+1}=r\left(t_{k}, x\right)
$$

For $0 \leq x<1$, the right first return path to $x$ based on $\left\{x_{n}\right\}, \mathcal{P}_{x}^{r}=\left\{s_{k}\right\}$, is defined analogously, and for each $x \in[0,1]$ the first return approach to $x$ based on $\left\{x_{n}\right\}, \mathcal{A}_{x}=\left\{u_{k}\right\}$, is defined recursively via

$$
u_{1}=r((0,1) \backslash\{x\}) \text {, and } u_{k+1}=r\left(B_{\left|x-u_{k}\right|}(x) \backslash\{x\}\right) .
$$


We say that $f:[0,1] \rightarrow \mathbb{R}$ is first return continuous from the left [right] at $x$ with respect to the trajectory $\left\{x_{n}\right\}$ provided

$$
\lim _{\substack{t \rightarrow x \\ t \in \mathcal{P}_{x}^{l}}} f(t)=f(x) \quad\left[\lim _{\substack{s \rightarrow x \\ s \in \mathcal{P}_{x}^{r}}} f(s)=f(x)\right] .
$$

We say that for any $x \in(0,1), f$ is first return continuous at $x$ with respect to the trajectory $\left\{x_{n}\right\}$ provided it is both left and right first return continuous at $x$ with respect to the trajectory $\left\{x_{n}\right\}$. We will adopt the convention of saying that $f$ is first return continuous at 0 with respect to $\left\{x_{n}\right\}$ provided it is first return continuous from the right there with respect to $\left\{x_{n}\right\}$, and adopt the symmetric convention concerning $x=1$. On the other hand, for $x \in[0,1]$ we say that $f$ is first return approachable at $x$ with respect to the trajectory $\left\{x_{n}\right\}$ provided

$$
\lim _{\substack{u \rightarrow x \\ u \in \mathcal{A}_{x}}} f(u)=f(x) .
$$

We say that $f$ is first return continuous [approachable] with respect to $\left\{x_{n}\right\}$ provided it is first return continuous [approachable] with respect to $\left\{x_{n}\right\}$ at each $x \in[0,1]$. Likewise, $f$ is said to be first return continuous [approachable] provided there exists a trajectory $\left\{x_{n}\right\}$ with respect to which $f$ is first return continuous [approachable].

\section{The Fine Tuning}

In [3] it was shown that a function $f:[0,1] \rightarrow \mathbb{R}$ belongs to Baire class one if and only if $f$ is first return recoverable. Here we wish to take a closer look at the proof of the "only if" direction as presented in [3] and observe that it can readily be modified to yield the following:

Lemma 1 If $f:[0,1] \rightarrow \mathbb{R}$ is Baire one, then there exists a trajectory $\left\{x_{n}\right\}$ such that for each $x \in[0,1]$, except the $x_{n}$ 's, $f$ is first return continuous at $x$ with respect to $\left\{x_{n}\right\}$.

Proof. With apologies to the reader for the inconvenience we are about to inflict, it seems that the most efficient method for indicating the proof is to note modifications which can be made to that given for Theorem 1 in [3]. We may proceed exactly as stated there until reaching the the conditions i) and ii) which are to be satisfied at the end of the $k^{\text {th }}$ stage. Replace these with the following two conditions: 
$\left.\mathrm{i}^{*}\right)$ If $x \in G_{\nu} \cap\left[\frac{i}{2^{n_{k}}}, \frac{i+1}{2^{n_{k}}}\right] \backslash\left\{x_{l}\right\}_{l=0}^{m_{k}}$ for some $i=0,1, \ldots, 2^{n_{k}-1}$ and some $G_{\nu} \in \mathcal{G}_{k}$, then there are points of $\left\{x_{l}\right\}_{l=0}^{m_{k}}$ in $\left[\frac{i}{2^{n_{k}}}, \frac{i+1}{2^{n_{k}}}\right]$ lying strictly to the left and to the right of $x$ and the closest such points to $x$ on the left and right of $x$ both belong to $G_{\nu}$.

ii*) If $x \in G_{\nu} \backslash\left\{x_{l}\right\}_{l=0}^{m_{k}}$ for some $G_{\nu} \in \mathcal{G}_{k-1}$ and $x_{l} \in \mathcal{P}_{x}^{l} \cup \mathcal{P}_{x}^{r}$ with $m_{k-1}<$ $l \leq m_{k}$, then $x_{l} \in G_{\nu}$.

We proceed exactly as in [3] at stage 1 , observing that $i^{*}$ ) and ii*) are trivially satisfied. We then assume that stages $1, \ldots, k$ have been successfully completed and follow the directions for selecting and ordering points at stage $k+1$ as given in [3]. Then instead of verifying i) and ii) at stage $k+1$ we verify $\left.i^{*}\right)$ and $\left.\mathrm{ii}^{*}\right)$ as follows.

To show that condition $\left.\mathrm{i}^{*}\right)$ holds, assume that $x \in G_{\nu} \cap\left[\frac{i}{2^{n} k+1}, \frac{i+1}{2^{n} k+1}\right] \backslash$ $\left\{x_{l}\right\}_{l=0}^{m_{k+1}}$ for some $i=0,1, \ldots, 2^{n_{k+1}-1}$ and some $G_{\nu} \in \mathcal{G}_{k+1}$ and let $J=$ $\left[\frac{i}{2^{n} k+1}, \frac{i+1}{2^{n} k+1}\right]$. Note that since we know that $x \notin\left\{x_{l}\right\}_{l=0}^{m_{k+1}}$, we know that $x$ is not an endpoint of $J$. Let $G_{\tau}$ be the unique element of $\mathcal{G}_{k+1}$ having the longest $\tau$ which intersects $J$. Note that $\tau$ is an extension of $\nu$. In stage $k+1$ the points in $J$ which are candidates to be added to the trajectory are the max and min of $J$ intersected with each of $G_{\tau}, G_{\left.\tau\right|_{m-1}}, G_{\left.\tau\right|_{m-2}}, \ldots, G_{\left.\tau\right|_{1}}$. Since we know that $x$ is not such a point, that $\tau$ is an extension of $\nu$, and that sequence $\left\{G_{\tau}, G_{\left.\tau\right|_{m-1}}, G_{\left.\tau\right|_{m-2}}, \ldots, G_{\left.\tau\right|_{1}}\right\}$ is monotonically increasing, it follows that points to the left and right of $x$ in $J$ are chosen at stage $k+1$, or earlier, and that the closest such chosen points to the left and to the right of $x$ will be from $G_{\nu}$, verifying $\left.\mathrm{i}^{*}\right)$.

To show that condition ii*) holds, assume that $x \in G_{\nu} \backslash\left\{x_{l}\right\}_{l=0}^{m_{k+1}}$ for some $G_{\nu} \in \mathcal{G}_{k}$ and that $x_{l} \in \mathcal{P}_{x}^{l} \cup \mathcal{P}_{x}^{r}$ with $m_{k}<l \leq m_{k+1}$. Without loss of generality, consider the case $x_{l} \in \mathcal{P}_{x}^{r}$. Let $I$ be the interval of length $1 / 2^{n_{k}}$ of the partition considered at stage $k$ which contains $x$. Then by condition $\left.\mathrm{i}^{*}\right)$ at stage $k, I$ also contains the nearest element of $\mathcal{P}_{x}^{r}$ restricted to $\left\{x_{l}\right\}_{l=0}^{m_{k}}$. Thus, $x_{l}$ must belong to $I$ as well. If $G_{\tau}$ is the unique element of $\mathcal{G}_{k}$ having the longest $\tau$ which intersects $I$, then $\tau$ is an extension of $\nu$. At stage $k+1$, the interval $I$ is subdivided into a finite collection $\mathcal{H}$ of intervals of length $1 / 2^{n_{k+1}}$. Let $J \in \mathcal{H}$ be such that $x_{l} \in J$. If $G_{\mu}$ is the unique element of $\mathcal{G}_{k+1}$ with the longest $\mu$ which intersects $J$, then either $\mu$ is an extension of $\tau$ and, consequently, of $\nu$, or $\mu$ has at least one entry equal to $k+1$. We know from condition $\mathrm{i}^{*}$ ) at stage $k+1$ that the closest element of $\left\{x_{n}\right\}_{n=0}^{m_{k+1}}$ to the right of $x$ belongs to $G_{\nu}$. Call this point $y$. Thus, by the order in which points from $J$ are appended to the trajectory at stage $k+1$, it follows that all such points either belong to $G_{\nu}$ or they are appended after $y$ is appended. Since $x_{l} \in \mathcal{P}_{x}^{r}$ restricted to $\left\{x_{n}\right\}_{n=0}^{m_{k+1}}$, it cannot be the case that $x_{l}$ is appended after 
$y$. Thus, $x_{l} \in G_{\nu}$, verifying ii*).

Using $\left.i^{*}\right)$ and $i i^{*}$ ), the proof is completed in the same manner that recoverability was demonstrated in [3] using i) and ii).

The next lemma is obvious, but we find it useful to state because we shall use it repeatedly in the proof of our main result.

Lemma 2 Let $D$ be a support set, $\left\{d_{j}\right\}_{j=0}^{\infty}$ any ordering of $D$, and $(a, b) \subset$ $[0,1]$. If $s=r(a, b)$ i.e., if $s$ is the first term of $\left\{d_{j}\right\}_{j=0}^{\infty}$ in $(a, b)$, then $s \in \mathcal{P}_{x}^{l}$ for all $x \in(s, b)$ and $s \in \mathcal{P}_{x}^{r}$ for all $x \in(a, s)$.

Let $f:[0,1] \rightarrow \mathbb{R}$. In stating and proving the following result, it is helpful to categorize the points in $x \in[0,1]$ into three types, depending upon how isolated $(x, f(x))$ is in the graph of $f$ : Type I points are those $x \in(0,1)$ for which $(x, f(x))$ is isolated on neither the left nor the right. (The point $x=0$ $[x=1]$ will be a type I point if $(0, f(0))[(1, f(1))]$ is not isolated on the right [left].) Type II points are those $x \in(0,1)$ for which $(x, f(x))$ is isolated on exactly one side. (The points 0 and 1 are never considered type II.) Type III points are those $x \in[0,1]$ for which $(x, f(x))$ is isolated.

Theorem 1 Let $f:[0,1] \rightarrow \mathbb{R}$ be a Baire one function. Then there exists a trajectory $\left\{x_{n}\right\}$ such that

A) $f$ is first return recoverable with respect to $\left\{x_{n}\right\}$.

$B)$ If $x$ is a type I point, then $f$ is first return continuous at $x$ with respect to $\left\{x_{n}\right\}$.

C) If $x$ is a type II point, then $f$ is first return approachable at $x$ with respect to $\left\{x_{n}\right\}$.

Proof. According to a well-known theorem of A. Gleyzal [4], there is an interval function $H$ which converges to $f$. This means that $H(I)$ is real valued for every closed interval $I \subseteq[0,1]$ and that for every $x \in[0,1]$ and every sequence $\left\{I_{n}\right\}$ of intervals converging to $x$, we have $\lim _{n \rightarrow \infty} H\left(I_{n}\right)=f(x)$, where by $\left\{I_{n}\right\}$ converging to $x$ we mean that $x$ belongs to each $I_{n}$ and the lengths, $\left|I_{n}\right|$, of the $I_{n}$ tend to 0 as $n$ tends to $\infty$.

Let $\left\{d_{k}\right\}_{k=0}^{\infty}$ be a trajectory satisfying the statement of Lemma 1 and let $D$ denote the range of this sequence. Note that all type II and III points must be in $D$. Our strategy for proving the theorem will be to produce a rearrangement $\left\{x_{n}\right\}_{n=0}^{\infty}$ of $\left\{d_{k}\right\}_{k=0}^{\infty}$ with respect to which the statements A), B), and C) will hold. As an aid in doing this, we shall be utilizing two methods or operations for selecting an element of $D$ from certain open intervals $(a, b)$. 
The "r-operation" selects the point $r(a, b)$ based on the trajectory $\left\{d_{k}\right\}$; that is, throughout this proof $r(a, b)$ will be the first element of $\left\{d_{k}\right\}$ which lies in $(a, b)$. The " $h$-operation" will only be applied in certain instances when $a$ and $b$ belong to $D \cup\{0,1\}$. If only one endpoint belongs to $D$, it is called the "preferred point" and if both belong to $D$, then the one with smaller subscript in $\left\{d_{k}\right\}$ is called "preferred." The only times when the $h$-operation will be applied are situations when (preferred point, $f$ (preferred point)) is not an isolated point of the graph of $f \mid[a, b]$ ( $f$, restricted to $[a, b]$.) When we apply the $h$-operation to such an interval, $h(a, b)$ will be a certain point $y \in(a, b) \cap D$ for which

$$
\max \{\mid f(\text { preferred point })-f(y)|,| \text { preferred point }-y \mid\}<(b-a) / 2 .
$$

We will often place an even more stringent requirement on how close $y$ is to the preferred point, but we shall always at least require (1).

We define a sequence $\left\{\delta_{k}\right\}_{k=0}^{\infty}$ by first choosing $0<\delta_{0}<1 / 3$ so small that if $I$ is any interval containing $d_{0}$ with $|I|<\delta_{0}$, then $\left|f\left(d_{0}\right)-H(I)\right|<1$. For $k \geq 1$ we choose a positive $\delta_{k}$ so that

- $\delta_{k}<\delta_{k-1} / 2$,

- $\delta_{k}<\min \left\{\left|d_{i}-d_{j}\right| / 2: i, j \leq k, i \neq j\right\}$,

- if $I$ is any interval containing $d_{j}, j \leq k$ and with $|I|<\delta_{k}$, then $\left|f\left(d_{j}\right)-H(I)\right|<1 /(k+1)$.

The symbols $\mathcal{R}_{x}, \mathcal{A}_{x}$, and $\mathcal{P}_{x}^{l}\left[\mathcal{P}_{x}^{r}\right]$ will designate the first return route, approach, and left [right] path to $x$ based on the trajectory $\left\{d_{k}\right\}$, while $\hat{\mathcal{R}}_{x}$, $\hat{\mathcal{A}}_{x}$, and $\hat{\mathcal{P}}_{x}^{l}\left[\hat{\mathcal{P}}_{x}^{r}\right]$ designate the corresponding items based on the trajectory $\left\{x_{n}\right\}$.

We shall define the rearrangement $\left\{x_{n}\right\}$ of $\left\{d_{k}\right\}$ inductively in stages. At the end of the $k^{\text {th }}$ stage we will have specified two natural numbers $m_{k}$ and $i_{k}$ and $\left\{x_{n}\right\}_{n=0}^{m_{k}}$ will have been chosen along with partitions $\left\{\mathcal{Q}_{i}\right\}_{i=0}^{i_{k}}$ of $[0,1]$, satisfying all of the following:

i) For each $i=0, \ldots, i_{k}$, all points of $\mathcal{Q}_{i}$ belong to $D \cup\{0,1\}$.

ii) For each $i=0, \ldots, i_{k}, \mathcal{Q}_{i}$ consists of $2^{i+1}+1$ points of $D \cup\{0,1\}$ and $\mathcal{Q}_{i}$ was formed by joining to $\mathcal{Q}_{i-1}$ one point from $D$ lying in the interior of each of the partition intervals of $\mathcal{Q}_{i-1}$, where $\mathcal{Q}_{-1}=\{0,1\}$. If $[a, b]$ is a partition interval of $\mathcal{Q}_{i-1}$, the newly selected point was chosen by applying either the $r$-operation or the $h$-operation to $(a, b)$.

iii) $\left\{d_{0}, d_{1}, \ldots, d_{k}\right\} \subseteq \mathcal{Q}_{i_{k}}$. 
iv) $\left\|\mathcal{Q}_{i_{k}}\right\|<\delta_{k}$, where $\left\|\mathcal{Q}_{i_{k}}\right\|$ denotes the mesh of $\mathcal{Q}_{i_{k}}$. (In particular, note that this condition implies that each partition interval of $\mathcal{Q}_{i_{k}}$ has at most one intersection with $\left\{d_{0}, d_{1}, \ldots, d_{k}\right\}$.)

v) For each $x \in[0,1]$ we have that if $y$ belongs to $\hat{\mathcal{P}}_{x}^{l} \cap\left(\mathcal{Q}_{i_{k}} \backslash \mathcal{Q}_{i_{k-1}}\right)$ $\left[\hat{\mathcal{P}}_{x}^{r} \cap\left(\mathcal{Q}_{i_{k}} \backslash \mathcal{Q}_{i_{k-1}}\right)\right]$, then either

a) $y \in \mathcal{P}_{x}^{l}\left[\mathcal{P}_{x}^{r}\right]$, or

b) there is a $j \in\{0, \ldots, k-1\}$ and a partition interval, say $J$, of $\mathcal{Q}_{i_{k-1}}$ containing $d_{j}$ and $x$, and $\left|f\left(d_{j}\right)-f(y)\right|<|J|$. Note that this implies

$$
\begin{aligned}
|f(x)-f(y)| \leq & |f(x)-H(J)|+\left|H(J)-f\left(d_{j}\right)\right|+ \\
& +\left|f\left(d_{j}\right)-f(y)\right| \\
\leq & |f(x)-H(J)|+1 / k+|J| .
\end{aligned}
$$

vi) if $x \in\left\{d_{0}, \ldots, d_{k-1}\right\}$ is a type II point and $y$ belongs to $\hat{\mathcal{A}}_{x} \cap\left(\mathcal{Q}_{i} \backslash \mathcal{Q}_{i-1}\right)$ for some $i \in\left\{i_{k-1}+1, i_{k-1}+2, \ldots, i_{k}\right\}$, then

$$
|f(x)-f(y)|<\left\|\mathcal{Q}_{i-1}\right\| .
$$

vii) if $x \in\left\{d_{0}, \ldots, d_{k-1}\right\}$ is a type I point and $y$ belongs to $\left(\hat{\mathcal{P}}_{x}^{l} \cup \hat{\mathcal{P}}_{x}^{r}\right) \cap$ $\left(\mathcal{Q}_{i} \backslash \mathcal{Q}_{i-1}\right)$ for some $i \in\left\{i_{k-1}+1, i_{k-1}+2, \ldots, i_{k}\right\}$, then

$$
|f(x)-f(y)|<\left\|\mathcal{Q}_{i-1}\right\| \text {. }
$$

Consider stage 0 in this process; that is, $k=0$. We start with $i=0$ and form the partition $\mathcal{Q}_{0}$ of $[0,1]$ by applying the $r$-operation to $(0,1)$. Suppose that $r(0,1)=d_{k_{0}}$, so that $\mathcal{Q}_{0}=\left\{0,1, d_{k_{0}}\right\}$. Note that $k_{0}$ will be 0 if $d_{0} \notin\{0,1\}$ and $k_{0}>0$ otherwise. In either case, $d_{0} \in \mathcal{Q}_{0}$ and $\left\|\mathcal{Q}_{0}\right\| \geq 1 / 2>\delta_{0}$. We begin the definition of our rearrangement $\left\{x_{n}\right\}$ of $\left\{d_{k}\right\}$ by ordering the points of $\mathcal{Q}_{0} \cap D$ from left to right. (Note that we will have defined at least $x_{0}$ and at most $x_{0}, x_{1}, x_{2}$. Further note that $d_{0}$ is among these.) We move on to $i=1$ and apply the $r$-operation to $\left(0, d_{k_{0}}\right)$ and $\left(d_{k_{0}}, 1\right)$ to obtain points $d_{k_{1}}$ and $d_{k_{2}}$ and set $\mathcal{Q}_{1}=\left\{0,1, d_{k_{0}}, d_{k_{1}}, d_{k_{2}}\right\}$. We then append $d_{k_{1}}$ and $d_{k_{2}}$ to $\left\{x_{n}\right\}$. If $\left\|\mathcal{Q}_{1}\right\|<\delta_{0}$, we set $i_{0}=1$ and let $m_{0}+1$ denote the number of points we have placed in $\left\{x_{n}\right\}$ to this point. Note that $2 \leq m_{0} \leq 4$. Observe that the conditions i)-iv) clearly hold for $k=0$ and that conditions vi) and vii) hold vacuously at this stage. Furthermore, condition v), part a) always holds due to Lemma 2 , terminating stage 0 . On the other hand, if $\left\|\mathcal{Q}_{1}\right\| \geq \delta_{0}$, we proceed inductively to define $\mathcal{Q}_{2}, \mathcal{Q}_{3}, \ldots$, always applying the $r$-operation to 
select new points and always appending the newly selected points to $\left\{x_{n}\right\}$ in a left to right manner. Clearly, $\mathcal{Q}_{i}$ will contain $d_{i}$ and, therefore, there is a first $i \equiv i_{0}$ for which $\left\|\mathcal{Q}_{i}\right\|<\delta_{0}$. We let $m_{0}+1$ denote the number of terms in $\left\{x_{n}\right\}$ to this point. Conditions i)-vii) are again easily seen to hold for $k=0$. Stage 0 is therefore completed.

Now assume that for some nonnegative integer $k$ stage $k$ has been completed; that is, $i_{k}$ and $m_{k}$ have been specified, the finite sequence $\left\{x_{n}\right\}_{n=0}^{m_{k}}$ has been designated, the partitions $\mathcal{Q}_{i}, i=0, \ldots, i_{k}$ have been chosen, and conditions i)-vii) are satisfied for $k$. We consider stage $k+1$. For each $i>i_{k}$ we shall be using the $r$-operation and the $h$-operation in picking new points for $\mathcal{Q}_{i}$.

We start with $i=i_{k}+1$. To every partition interval of $\mathcal{Q}_{i_{k}}$ neither of whose endpoints belong to $\left\{d_{0}, \ldots, d_{k}\right\}$ we apply the $r$ operation to select a new point to be put in the partition $\mathcal{Q}_{i}$. Next, consider the partition interval(s) of $\mathcal{Q}_{i_{k}}$ which have some $d_{j}, j=0, \ldots, k$ as an endpoint. Note that by condition (iv), each interval contains at most one such $d_{j}$. If $\left(d_{j}, f\left(d_{j}\right)\right)$ is isolated in the graph of $f$ from within the partition interval, then apply the $r$ operation to that interval to select a point to be added to $\mathcal{Q}_{i}$. Do this for each $j=0, \ldots, k$, where appropriate. Designate the set of all points selected in this manner by $R_{i}$. Return to each $d_{j}, j=0, \ldots, k$ which is a type I or II point. For each such $d_{j}$ there are either one or two partition intervals of $\mathcal{Q}_{i_{k}}$ having $d_{j}$ as an endpoint and from which no point has yet been selected for $\mathcal{Q}_{i}$. Note that for each such $d_{j}$ and each such interval $J$ the graph of $f$ is not isolated on the side of $d_{j}$ containing $J$. Therefore, since $D$ satisfies Lemma 1 , it is also not isolated from $f \mid D$. With $d_{j}$ as the "preferred point", we apply the $h$-operation to each such partition interval, say $J$, containing $d_{j}$, finding a point $y \in D \cap J$ such that

$$
\begin{gathered}
\left|y-d_{j}\right|<\min \left\{|J| / 2, \operatorname{dist}\left\{d_{j}, R_{i}\right\}\right\}, \text { and } \\
\left|f(y)-f\left(d_{j}\right)\right|<|J| / 2 .
\end{gathered}
$$

Let's call the set of all of the points selected using the $h$-operation in this manner $H_{i}$. Then $\mathcal{Q}_{i}=\mathcal{Q}_{i_{k}} \cup R_{i} \cup H_{i}$. Next, moving from left to right, we append the points of $H_{i}$ to $\left\{x_{n}\right\}_{n=1}^{m_{k}}$; then, moving from left to right, we append the points of $R_{i}$. If $\left\|\mathcal{Q}_{i}\right\|<\delta_{k+1}$ and $d_{k+1} \in \mathcal{Q}_{i}$, we terminate stage $k+1$ here, setting $i_{k+1}=i$ and $m_{k+1}+1$ equal to the number of terms in $\left\{x_{n}\right\}$ to date. Conditions i)-vii) hold for $k+1$. On the other hand, if $\left\|\mathcal{Q}_{i}\right\| \geq \delta_{k+1}$ or $d_{k+1} \notin \mathcal{Q}_{i}$, then we repeat the procedure until both conditions are satisfied. Conditions i)-vii) are seen to hold for $k+1$ and the inductive construction of the rearrangement $\left\{x_{n}\right\}$ is complete. However, we must pause to verify that we will indeed encounter a $j_{k}$ for which both $\left\|\mathcal{Q}_{i_{k}+j_{k}}\right\|<\delta_{k+1}$ and $d_{k+1} \in \mathcal{Q}_{i_{k}+j_{k}}$. 
To verify both parts of this claim simultaneously, we shall show that for each nonnegative integer $m$ there is a $j_{m}$ for which $\mathcal{Q}_{i_{k}+j_{m}}$ contains $\left\{d_{0}, \ldots, d_{m}\right\}$. Clearly this is true for $m=0$. Assume it is true for some nonnegative integer $m$; that is, assume that $\mathcal{Q}_{i_{k}+j_{m}}$ contains $\left\{d_{0}, \ldots, d_{m}\right\}$. If $d_{m+1} \in \mathcal{Q}_{i_{k}+j_{m}}$, we are done. So, suppose that $d_{m+1} \notin \mathcal{Q}_{i_{k}+j_{m}}$. Then there is a unique partition interval $J$ of $\mathcal{Q}_{i_{k}+j_{m}}$ which contains $d_{m+1}$. If the $r$-operation is applied to $J$, then $d_{m+1}$ must be selected for membership in $\mathcal{Q}_{i_{k}+j_{m}+1}$ and we are done. However, if the $h$-operation is applied to $J$, then $d_{m+1}$ might not be selected for membership in $\mathcal{Q}_{i_{k}+j_{m}+1}$. Suppose that it is not. The fact that the $h$-operation was applied implies that $J$ has some $d_{j_{0}}$, $0 \leq j_{0} \leq k$, as an endpoint with other endpoint not in $\left\{d_{j}: j=0, \ldots, k\right\}$, and $\left(d_{j_{0}}, f\left(d_{j_{0}}\right)\right)$ is not isolated in the graph of $f \mid J$. The point $y_{1}$ selected via the $h$-operation will have the property that $\left|y_{1}-d_{j_{0}}\right|<|J| / 2$. Note that if $\left|d_{m+1}-d_{j_{0}}\right|>\left|y_{1}-d_{j_{0}}\right|$ then in forming $\mathcal{Q}_{i_{k}+j_{m}+2}$ the $r$-operation will be applied to the unique partition interval of $\mathcal{Q}_{i_{k}+j_{m}+1}$ containing $d_{m+1}$ and thus it will be selected. On the other hand if $\left|d_{m+1}-d_{j_{0}}\right|<\left|y_{1}-d_{j_{0}}\right|$, then the $h$-operation will be applied instead, yielding a point $y_{2}$ which might not equal $d_{m+1}$. This process can continue, but notice that the $y_{l}$ 's are converging toward $d_{j_{0}}$ and hence we will encounter a situation when $\left|d_{m+1}-d_{j_{0}}\right|>\left|y_{l}-d_{j_{0}}\right|$. Once this situation is encountered, the $r$-operation will be applied and $d_{m+1}$ will have to be selected for membership in $\mathcal{Q}_{i_{k}+j_{m}+l+1}$. Thus, we could set $j_{m+1}=j_{m}+l+1$ and our claim is established.

Next, note that $\left\{x_{n}\right\}_{n=0}^{\infty}$ is, indeed, an ordering of $D$; that is, it is a rearrangement of $\left\{d_{k}\right\}_{k=0}^{\infty}$, this following from condition iii). Now we must show that statements A), B) and C) are valid with respect to this trajectory $\left\{x_{n}\right\}$.

First, note that if $x$ is a type III point in $D$, then $x$ is an element in $\left\{x_{n}\right\}$ and thus $f$ is first return recoverable at $x$ with respect to $\left\{x_{n}\right\}$.

Next, suppose that $x$ is a type II point in $D$; say $x=d_{k}$. Let $\epsilon>0$ be given and choose $N \geq m_{k+1}$ so large that $\left\|\mathcal{Q}_{N}\right\|<\epsilon$. Then from vi) we see that every $x_{n} \in \hat{\mathcal{A}}_{x}$ with $x_{n} \notin \mathcal{Q}_{N}$ satisfies $\left|f(x)-f\left(x_{n}\right)\right|<\epsilon$, indicating that $f$ is first return approachable at $x$ with respect to $\left\{x_{n}\right\}$.

Similarly, suppose that $x$ is a type I point in $D$; say $x=d_{k}$. Let $\epsilon>0$ be given and choose $N \geq m_{k+1}$ so large that $\left\|\mathcal{Q}_{N}\right\|<\epsilon$. Then from vii) we see that every $x_{n} \in \hat{\mathcal{P}}_{x}^{l} \cup \hat{\mathcal{P}}_{x}^{r}$ with $x_{n} \notin \mathcal{Q}_{N}$ satisfies $\left|f(x)-f\left(x_{n}\right)\right|<\epsilon$, indicating that $f$ is first return continuous at $x$ with respect to $\left\{x_{n}\right\}$.

To complete the proof of the theorem, it only remains to show that $f$ is first return continuous at each $x \in[0,1] \backslash D$. Consider such an $x$ and let $\left\{u_{l}\right\}$ be any sequence from $\hat{\mathcal{P}}_{x}^{l} \cup \hat{\mathcal{P}}_{x}^{r}$ converging to $x$. We use condition $\left.\mathrm{v}\right)$ to break $\left\{u_{l}\right\}$ into two subsequences. (We shall proceed, without loss of generality, 
assuming that both subsequences are, indeed, infinite.) We let $\left\{v_{j}\right\}_{j=1}^{\infty}$ denote the subsequence whose terms belong to $\mathcal{P}_{x}^{l} \cup \mathcal{P}_{x}^{r}$ and let $\left\{v_{m}\right\}_{m=1}^{\infty}$ denote the remaining subsequence. From Lemma 1 we know that $\lim _{j \rightarrow \infty} f\left(v_{j}\right)=f(x)$.

Next, let $\epsilon>0$ and choose $\delta>0$ such that if $I$ is any interval containing $x$ with $|I|<\delta$, then $|H(I)-f(x)|<\epsilon / 3$. Let $K \in \mathbb{N}$ be so large that $1 / K<\epsilon / 3$ and $\left\|\mathcal{Q}_{i_{K}}\right\|<\min \{\delta, \epsilon / 3\}$. Choose $M \in \mathbb{N}$ so large that for all $m>M$ we have $\left|v_{m}-x\right|<\min \left\{\left|x-d_{k}\right|: d_{k} \in \mathcal{Q}_{i_{K}}\right\}$. Consider any $m>M$. Choose the first $k$ so that $v_{m} \in \mathcal{Q}_{i_{k}}$ and observe that $k>K$. From condition v) at stage $k$, it follows that there is some $j \in\{0,1, \ldots k-1\}$ such that $d_{j}$ and $x$ belong to a partition interval, say $J$, of $\mathcal{Q}_{i_{k-1}}$ and

$$
\left|f(x)-f\left(v_{m}\right)\right|<|f(x)-H(J)|+1 / k+|J|<\frac{\epsilon}{3}+\frac{\epsilon}{3}+\frac{\epsilon}{3}=\epsilon,
$$

showing that $f$ is first return continuous at $x$ with respect to $\left\{x_{n}\right\}$ and completing the proof of the theorem.

We remark that not just any trajectory $\left\{d_{j}\right\}$ with respect to which $f$ is first return recoverable can be rearranged to form a trajectory yielding statements A), B), and C) of Theorem 1. Indeed, an example is given in [1] of a Baire one $f$ and a trajectory $\left\{d_{j}\right\}$ with respect to which $f$ is first return approachable, but for which, given any ordering $\left\{x_{n}\right\}$ of $\left\{d_{j}\right\}$, the set of points at which $f$ is not first return continuous with respect to $\left\{x_{n}\right\}$ is uncountable (in fact, perfectly dense in $[0,1]$ ), whereas it is well-known that at most countably many points can fail to be type III points.

The authors wish to thank the referee for carefully reading the original manuscript and offering suggestions which have resulted in an improved exposition.

\section{References}

[1] U. B. Darji, M. J. Evans, and P. D. Humke, First return approachability, J. Math. Anal. and Appl. (to appear).

[2] U. B. Darji, M. J. Evans, and R. J. O'Malley, First return path systems: differentiability, continuity, and orderings, Acta Math. Hungar. 66 (1995), 83-103.

[3] U. B. Darji, M. J. Evans, and R. J. O'Malley, A first return characterization of Baire one functions, Real Anal. Exch. 19 (1993-94), 510-515.

[4] A. Gleyzal, Interval-functions, Duke Math. J. 8 (1941), 223-230. 